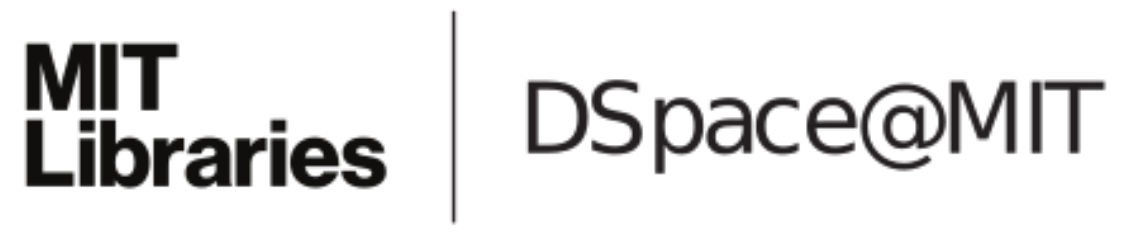

\author{
MIT Open Access Articles
}

Hydrogen-Enhanced Vacancy Diffusion in Metals

The MIT Faculty has made this article openly available. Please share how this access benefits you. Your story matters.

As Published: 10.1021/ACS.JPCLETT.0C01798

Publisher: American Chemical Society (ACS)

Persistent URL: https://hdl.handle.net/1721.1/133223

Version: Final published version: final published article, as it appeared in a journal, conference proceedings, or other formally published context

Terms of Use: Article is made available in accordance with the publisher's policy and may be subject to US copyright law. Please refer to the publisher's site for terms of use. 


\title{
Hydrogen-Enhanced Vacancy Diffusion in Metals
}

\author{
Jun-Ping Du, W. T. Geng, Kazuto Arakawa, Ju Li,* and Shigenobu Ogata*
}

Cite This: J. Phys. Chem. Lett. 2020, 11, 7015-7020

Read Online

ABSTRACT: Vacancy diffusion is fundamental to materials science. Hydrogen atoms bind strongly to vacancies and are often believed to retard vacancy diffusion. Here, we use a potential-of-mean-force method to study the diffusion of vacancies in $\mathrm{Cu}$ and $\mathrm{Pd}$. We find $\mathrm{H}$ atoms, instead of dragging, enhance the diffusivity of vacancies due to a positive hydrogen Gibbs excess at the saddle-point: that is, the migration saddle attracts more $\mathrm{H}$ than the vacancy ground state, characterized by an activation excess $\Gamma_{\mathrm{H}}^{\mathrm{m}} \approx 1 \mathrm{H}$, together with also-positive migration activation volume $\Omega^{\mathrm{m}}$ and activation entropy $S^{\mathrm{m}}$. Thus, according to the Gibbs adsorption isotherm generalized to the activation path, a higher $\mu_{\mathrm{H}}$ significantly lowers the migration free-energy barrier. This is verified by $a b$ initio grand canonical Monte Carlo simulations and direct molecular dynamics simulations. This trend is believed to be generic for migrating dislocations, grain boundaries, and so on that also have a higher capacity for attracting $\mathrm{H}$ atoms due to a positive activation volume at the migration saddles.
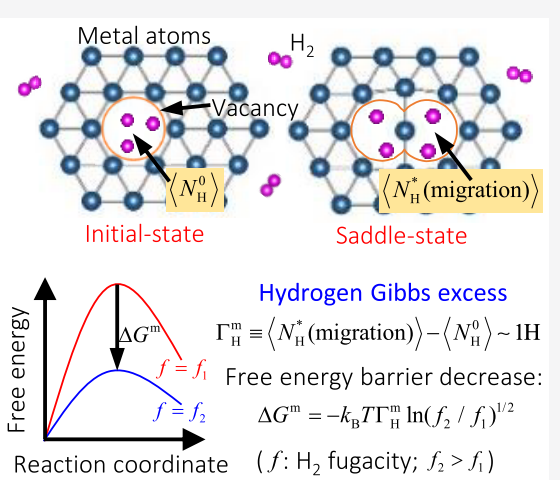

$\mathrm{H}$ ydrogen can either be an intentional constituent or unwelcome impurity in materials. ${ }^{1}$ As a deliberate ingredient, a high concentration of $\mathrm{H}$ can cause the metal$\mathrm{H}$ systems to function as energy-storage media, ${ }^{2,3}$ sensors, ${ }^{4}$ or catalysts. ${ }^{5,6}$ Hydrogen is also notorious for degrading the mechanical properties of metals, in the form of hydrogen embrittlement $^{7-9}$ and hydrogen blistering/bubbling. ${ }^{10-12}$ Hydrogen (tritium) is inevitably introduced into metals during forming, finishing operations, and radiation and environmental exposures, in for example nuclear fission and fusion power plant components. Due to the high mobility even at room temperature, $\mathrm{H}$ atoms can reach and achieve thermodynamic equilibrium quickly around the structural defects in metals, ${ }^{1}$ such as vacancies, dislocations, and grain boundaries, changing their stability and mobility.

Transition-state theory ${ }^{13}$ (TST), also referred to as activated-complex theory, is used to model the rate of system jumping from one free-energy minimum to another. The stress and temperature dependence of the jump rate $(R)$ are characterized by the activation volume $\left(\Delta V^{*}\right)$ and the activation entropy $\left(\Delta S^{*}\right)$, respectively, that is

$$
R=\nu_{0} \exp \left(-\frac{\Delta U^{*}+P \Delta V^{*}-T \Delta S^{*}}{k_{\mathrm{B}} T}\right)
$$

where $\nu_{0}$ is the attempt frequency, $\Delta U^{*}$ is the potential energy difference between the reactants (initial state) and the activated transition-state complex (saddle state, often denoted by the superscript $*), P$ is the stress, $T$ is the temperature, and $k_{\mathrm{B}}$ is Boltzmann's constant. The diffusive transport of metal atoms $(\mathrm{M})$ in crystalline materials is dominated by the vacancy-exchange mechanism ${ }^{14,15}$ and was studied based on TST using computational techniques for searching the minimum energy pathway (MEP). A key question is how to treat hydrogen segregation, i.e., do we consider the number of hydrogen $\left(N_{\mathrm{H}}\right)$ in the activated complex to be constant during the migration process, or do we allow it to vary? A careful examination of TST in the statistical sense indicates that it should be the latter. That is, we should allow $N_{\mathrm{H}}$ to be adjusted also (in the statistical kinetics sense), giving rise to a migration activation excess $\Gamma_{\mathrm{H}}^{\mathrm{m}} \equiv\left\langle\Delta N_{\mathrm{H}}^{*}\right.$ (migration) $\rangle$ in the notation of the Gibbs adsorption isotherm

$$
\mathrm{d} G^{\mathrm{m}}=-\Gamma_{\mathrm{H}}^{\mathrm{m}} \mathrm{d} \mu_{\mathrm{H}}+\Omega^{\mathrm{m}} \mathrm{d} P-S^{\mathrm{m}} \mathrm{d} T
$$

for the migration free-energy barrier $G^{\mathrm{m}}$, parallel to the definition of the migration activation volume $\Omega^{\mathrm{m}} \equiv \Delta V^{*}$. (migration) $\equiv V_{\text {saddle }}\left(T, P, \mu_{\mathrm{H}}\right)-V_{\text {initial }}\left(T, P, \mu_{\mathrm{H}}\right)$ and activation entropy $S^{\mathrm{m}} \equiv \Delta S^{*}($ migration $) \equiv S_{\text {saddle }}\left(T, P, \mu_{\mathrm{H}}\right)-$ $S_{\text {initial }}\left(T, P, \mu_{\mathrm{H}}\right)$.

Hydrogen atoms tend to segregate in the vacancy, often offset from the site center. This behavior of the $\mathrm{H}$-vacancy complex has been explained by the effective medium theory, ${ }^{16}$ which indicates that the energy needed to introduce $\mathrm{H}$ atoms into a background electron density, known as the embedding energy, achieves an optimal value at a preferred background electron density; thus, $\mathrm{H}$ atoms seek spots with such preferred electron density on the outer surface and inner interstices of the metal. The mobility of $\mathrm{H}$-vacancy complexes has been

Received: June 10, 2020

Accepted: July 30, 2020

Published: July 30, 2020 
studied using the TST and first-principles calculations, often adopting $\mathrm{M}-\mathrm{V}^{0}$ distance as the reaction coordinate, where $\mathrm{V}^{0}$ denotes the original vacancy site. Previous first-principles calculations based on density functional theory (DFT) have indicated that the $\mathrm{H}$ atoms trapped by vacancies can substantially increase the energy barrier to metal-vacancy exchange, ${ }^{17,18}$ if $N_{\mathrm{H}}$ is fixed. Particularly, the ratio of the vacancy diffusion coefficient with and without $\mathrm{H}$ in a $\mathrm{Ni}-\mathrm{H}$ system, $D_{\mathrm{NiH}}^{\mathrm{V}} / D_{\mathrm{Ni}}^{\mathrm{V}}$, decreases dramatically with increasing $\mathrm{H}$ concentration, and the ratio can be as low as $10^{-4}$ at $c_{\mathrm{H}}=0.1$ ( $\mathrm{H}$-concentration in atomic ratio) and $700 \mathrm{~K}$ based on the DFT calculations. ${ }^{17}$ This is based on the argument that during the rare but violent multiphonon-collisional atomic dynamics of thermally activated processes at picosecond time scale, there is sufficient time for the $N_{\mathrm{H}}$ hydrogens surrounding the activated complex to adjust their positions but no time for other hydrogens far way to arrive and have an effect during this migration, which is certainly true. Such a fixed- $N_{\mathrm{H}}$ argument, however, neglects the fact that there is not just one path but many possible activation paths that could lead to vacancy exchange. Indeed, the optimal $N_{\mathrm{H}}^{*}$ (migration) that minimizes the absolute Helmholtz free energy of the vacancy-exchange saddle-point is unlikely to be the $N_{\mathrm{H}}$ that optimizes the absolute Helmholtz free energy of the ground state, when this vacancy is immersed in a large hydrogenated crystal bath. So one can imagine the configuration having $N_{\mathrm{H}}^{*}$ instead of $N_{\mathrm{H}}$ hydrogen prepositioned in the vacancy (which is certainly not optimal and thus an excited configurational state), which then undergoes multiphonon-collision actuated atomic dynamics. By going through this excited precursor state with a chemical excess different from the ground-state configuration, one can then end up with the lowest absolute energy activated complex and therefore the largest TST rate contribution, more than the $N_{\mathrm{H}}=N_{\mathrm{H}}^{0}$ path, where superscript 0 is used to denote the ground-state optimum. In above, $N_{\mathrm{H}}^{0}$ and $N_{\mathrm{H}}^{*}$ are integers. By summing up all path contributions with various $N_{\mathrm{H}}$, one can derive a Gibbs free-energy barrier, with $\Gamma_{\mathrm{H}}^{\mathrm{m}} \equiv \Gamma_{\mathrm{H}}^{*}-\Gamma_{\mathrm{H}}^{0} \equiv$ $\left\langle N_{\mathrm{H}}^{*}\right.$ (migration) $\rangle-\left\langle N_{\mathrm{H}}^{0}\right\rangle$, where $\Gamma_{\mathrm{H}}^{*}$ and $\Gamma_{\mathrm{H}}^{0}$ are statistical averages in the sense of the Gibbs adsorption isotherm ${ }^{19,20}$ (Figure 1), $\mathrm{d} G=-\Gamma_{\mathrm{H}} \mathrm{d} \mu_{\mathrm{H}}+\Omega \mathrm{d} P-S \mathrm{~d} T$ for the ground- and saddle-state respectively, and each can be a noninteger. Unlike traditional surface/interfacial excess, here we choose not to normalize mass excess versus an area (or length), because the vacancy is not an extended defect.

We would like to show conceptually that $\Gamma_{\mathrm{H}}^{\mathrm{m}}$ is usually positive, just like the migration activation volume $\Omega^{\mathrm{m}}$ (symbolizing dilation or extra free volume ${ }^{21}$ during migration) and activation entropy $S^{\mathrm{m}}>0$ (symbolizing vibrational mode softening, as indicated by the Meyer-Neldel compensation rule ${ }^{22,23}$ with positive $T_{\mathrm{MN}}$ ). We can define a scalar reaction coordinate $\xi$ based on the $\mathrm{M}-\mathrm{V}^{0}$ distance $(d): \xi \equiv 1-d / d_{\max }$. $\xi=0,1$ denotes the initial and final states, both perfect vacancies, while $\xi=0.5$ denotes the saddle-point state, which is known both experimentally and from first-principles calculations to have a larger free volume and is vibrationally softer on average even after the imaginary-frequency phonon mode along the MEP is taken out. As illustrated in Figure 1, as M moves half way between the $\mathrm{V}^{0}$ and $\mathrm{V}^{1}$ sites, there is crowding between $\mathrm{M}$ and metal atoms, forcing an outward expansion of those metal atoms, enlarging the total volume $\left(\Omega^{\mathrm{m}}>0\right)$. But also, both $\mathrm{V}^{0}$ and $\mathrm{V}^{1}$ site centers are effectively vacated, leaving significantly larger "internal surface areas" for hydrogen atoms to experience the optimal background electron density. It is

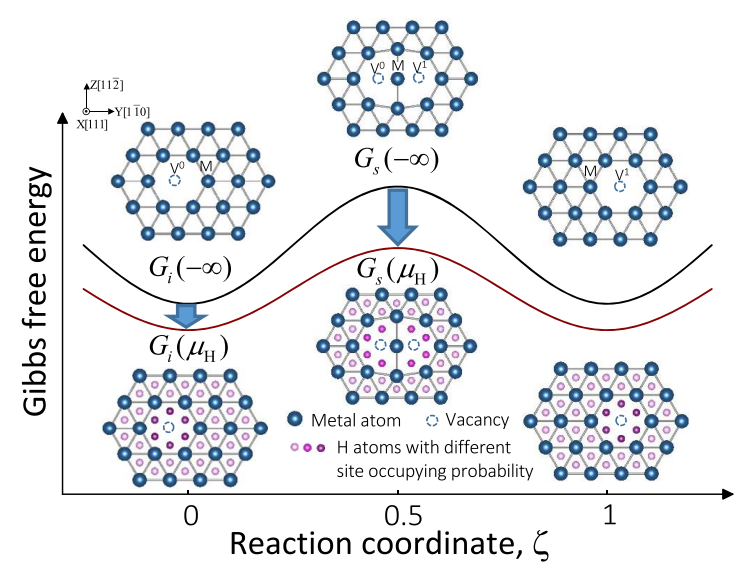

Figure 1. Schematics of Gibbs free-energy changes of the vacancy diffusion as a function of reaction coordinate in pure metals (black line) and a metal-H system (dark red line) under a specific $\mathrm{H}$ concentration corresponding to a specific chemical potential of $\mathrm{H}$ $\left(\mu_{\mathrm{H}}\right)$. The blue balls in the insets are metal atoms on one (111) plane with one vacancy. The small balls in the insets are $\mathrm{H}$ atoms in the interstitial sites colored by the site occupying probability. The metal atom, labeled by $\mathrm{M}$, moves from the final vacancy site $\left(\mathrm{V}^{1}\right.$ at $\left.\xi=1\right)$ to the initial vacancy site $\left(\mathrm{V}^{0}\right.$ at $\left.\xi=0\right)$, while at the saddle-point state ( $\xi$ $=0.5$ ), both $\mathrm{V}^{0}$ and $\mathrm{V}^{1}$ are effectively vacant and are able to trap more $\mathrm{H}$ atoms overall.

therefore natural to suspect $\Gamma_{\mathrm{H}}^{\mathrm{m}}>0$ due to the larger $\mathrm{H}$ trapping capacity of the activated complex. Thus, if the activation excess $\Gamma_{\mathrm{H}}^{\mathrm{m}}=1 \mathrm{H}$, for every $10 \times$ increase in equivalent hydrogen gas partial pressure (fugacity) $P_{\mathrm{H}_{2}}$, we would predict a $(\sqrt{10} \approx 3.162 \times)$ increase in vacancy mobility, regardless of temperature. Note that generally speaking, $\Gamma_{\mathrm{H}}^{\mathrm{m}}$ is temperature-dependent (see Secs. S2 and S8 in the Supporting Information). We term such hydrogen-dependent defect mobility the "hydrogen lubrication" effect, which could be operative for dislocations as well as grain boundaries.

Here, we performed the potential-of-mean-force ${ }^{24}$ (PMF) calculations, $a b$ initio grand canonical Monte Carlo (GCMC) simulations, and direct molecular dynamics (MD) simulations, to study the vacancy diffusivity under different H-concentrations, exploring the correlation between the diffusivity of the vacancy and the chemical composition variation of $\mathrm{H}$ along the vacancy diffusion pathway. Moreover, we choose $\mathrm{Cu}-\mathrm{H}$ and $\mathrm{Pd}-\mathrm{H}$ as prototype systems, since a large amount of $\mathrm{H}$ can be incorporated in the metals via high $\mathrm{H}_{2}$ pressure ${ }^{25}$ or an electrodeposition process. ${ }^{26}$ For example, the $\mathrm{H}$ fraction in $\mathrm{Pd}$ ( $\mathrm{H}$ to metal atomic ratio) can reach 0.7 under a $\mathrm{H}_{2}$ pressure of $2 \mathrm{GPa}^{27}$ and 0.02 through electrodeposition. ${ }^{28}$

To clarify the effect of chemical composition variation along the vacancy diffusion pathway, we computed the free-energy barrier by the integral of the time-averaged force on the jumping metal atom over the reaction coordinates of the vacancy diffusion from the initial energy minimum to the saddle state based on the PMF method with the embeddedatom-method (EAM) potentials ${ }^{29-31}$ (see Secs. S3 and S9 in the Supporting Information). On the basis of the TST, the jump frequency $(R)$ is given by $R=v_{0} \exp \left(-G^{\mathrm{m}} / k_{\mathrm{B}} T\right)$, where $v_{0}$ is the attempt frequency, and $G^{\mathrm{m}}$ is the free-energy barrier for the vacancy jump as computed using the PMF simulations. The uncorrelated diffusion coefficient of vacancy is estimated by ${ }^{32} D^{\mathrm{V}}=a_{0}^{2} R$, where $a_{0}$ is the lattice constant. Assuming the same $v_{0}$ for the pure metal and metal-H systems, the vacancy 
diffusion coefficient ratios with and without $\mathrm{H}$ at $870 \mathrm{~K}$ from the PMF simulation are computed using $D_{\mathrm{MH}}^{\mathrm{V}} / D_{\mathrm{M}}^{\mathrm{V}}=$ $\exp \left(-\Delta G^{\mathrm{m}} / k_{\mathrm{B}} T\right)$ and shown in Figure $2(\mathrm{a}, \mathrm{b})$, where $\mathrm{M}=$
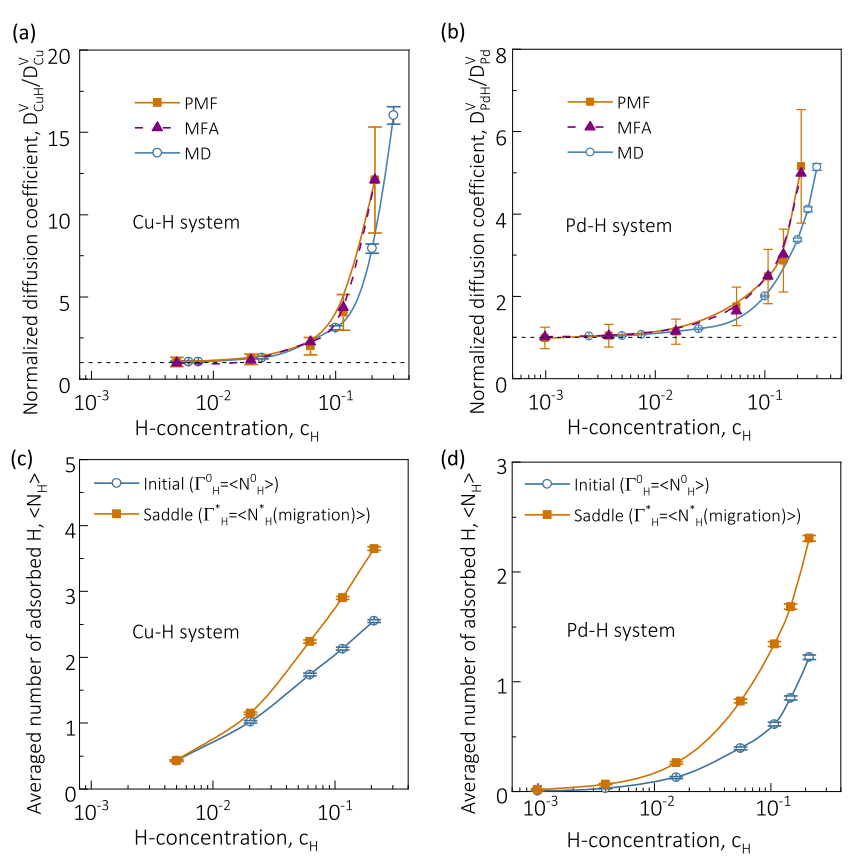

Figure 2. Vacancy diffusivity and the chemical composition variation of $\mathrm{H}$ along the vacancy diffusion pathway in a metal- $\mathrm{H}$ system. (a) and (b) manifest the ratio of the vacancy diffusion coefficient in the metal- $\mathrm{H}$ system to that in the pure metal system at $870 \mathrm{~K}$ computed from the potential-of-mean-force (PMF) method, an analytic expression under a mean field approximation (MFA) and direct MD method. (c) and (d) show the number of adsorbed $\mathrm{H}$ atoms at the initial and the saddle states as computed from the PMF method. The error bars in $(a-d)$ are standard error of the data.

$\mathrm{Cu}$ or $\mathrm{Pd}$, and $\Delta G^{\mathrm{m}}=G^{\mathrm{m}}-G_{0}^{\mathrm{m}}$ is the difference of free-energy barrier between the $\mathrm{M}-\mathrm{H}$ system $\left(G^{\mathrm{m}}\right)$ and pure $\mathrm{M}$ system $\left(G_{0}^{\mathrm{m}}\right)$. From Figure $2(\mathrm{a}, \mathrm{b})$, for $\mathcal{c}_{\mathrm{H}}>0.01$, the PMF surprisingly predicts a diffusivity substantially greater than that in pure $\mathrm{Cu}$ and $\mathrm{Pd}$ (e.g., $D_{\mathrm{CuH}}^{\mathrm{V}} / D_{\mathrm{Cu}}^{\mathrm{V}} \approx 12$ at $\left.c_{\mathrm{H}}=0.2\right)$. Next, we computed the number of $\mathrm{H}$ atoms adsorbed in the initial $\left(\Gamma_{\mathrm{H}}^{0} \equiv\left\langle N_{\mathrm{H}}^{0}\right\rangle\right)$ and the saddle $\left(\Gamma_{\mathrm{H}}^{*} \equiv\left\langle N_{\mathrm{H}}^{*}(\right.\right.$ migration $\left.\left.)\right\rangle\right)$ states. In an FCC lattice with a single vacancy, eight first-nearest-neighbor tetragonal $[1 \mathrm{NN}(\mathrm{T})]$ and six second-nearest-neighbor octahedral $[2 \mathrm{NN}(\mathrm{O})]$ interstitial sites are located near the vacancy center of $\mathrm{V}^{0}$ and $\mathrm{V}^{1}$. According to the interaction energy between the $\mathrm{H}$ atom and the vacancy, the $\mathrm{H}$ atoms within $2 \mathrm{NN}(\mathrm{O})$ from the vacancy center of $\mathrm{V}^{0}$ are counted as $\Gamma_{\mathrm{H}}^{0}$ in the initial state (see Sec. S4 and Figures S7 and S8 in the Supporting Information). Whereas, in the saddle-point state, the $\mathrm{H}$ atoms within $2 \mathrm{NN}(\mathrm{O})$ from the vacancy centers of both $\mathrm{V}^{0}$ and $\mathrm{V}^{1}$ can be counted as $\Gamma_{\mathrm{H}}^{*}$. The results of $\Gamma_{\mathrm{H}}^{0}$ and $\Gamma_{\mathrm{H}}^{*}$ are shown in Figure 2(c,d), respectively. We can see that $\Gamma_{\mathrm{H}}^{*}$ is more than $\Gamma_{\mathrm{H}}^{0}$ but less than the twice of that $\left(2 \Gamma_{\mathrm{H}}^{0}>\Gamma_{\mathrm{H}}^{*}>\Gamma_{\mathrm{H}}^{0}\right)$. This means that while the additional $\mathrm{H}$ atoms are adsorbed near $\mathrm{V}^{1}$ at the saddle-point state, some $\mathrm{H}$ atoms are also squeezed out from $\mathrm{V}^{0}$. But, the total number of the adsorbed $\mathrm{H}$ in the saddle state is more than that in the initial state.

$A b$ initio GCMC simulation is a reliable method to explore the surface structures and compositions. ${ }^{33}$ To further validate the generally positive activation excess $\Gamma_{\mathrm{H}}^{\mathrm{m}} \equiv \Gamma_{\mathrm{H}}^{*}-\Gamma_{\mathrm{H}}^{0} \approx 1 \mathrm{H}$ in vacancy migration, we performed $a b$ initio GCMC simulations in the $\mathrm{Cu}-\mathrm{H}$ and the $\mathrm{Pd}-\mathrm{H}$ systems to compute $\Gamma_{\mathrm{H}}^{0}$ and $\Gamma_{\mathrm{H}}^{*}$ in the ground and the saddle states of vacancy migration, respectively (see Sec. S5 in Supporting Information). To reduce the computational cost, the thermal movements of metal atoms and the thermal expansion of the lattice are ignored in most of the simulations, while the $\mathrm{H}$ atoms are only moved and inserted/deleted in the region of the "internal surface" of the vacancy (Figure 3(a)) under a specific $\mu_{\mathrm{H}}$ and a (a)

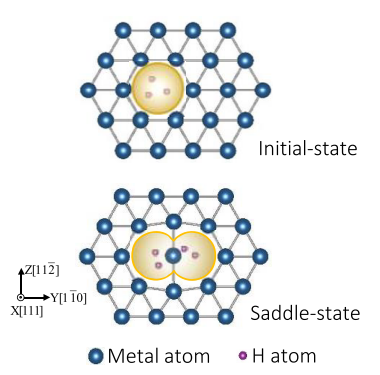

(c)

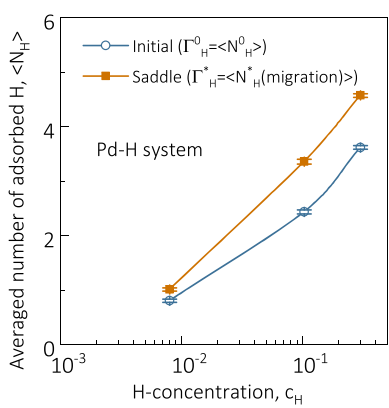

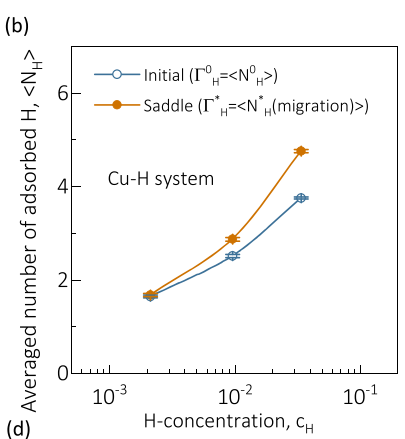

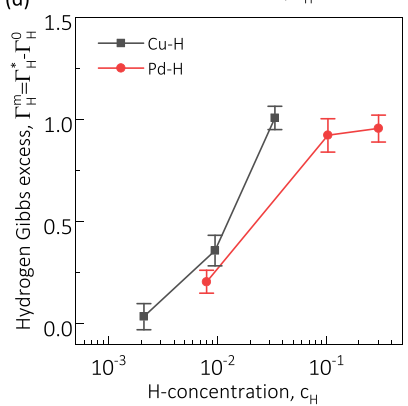

Figure 3. (a) Schematics of the initial and the saddle states used in $a b$ initio GCMC simulations. The blue balls are metal atoms on one (111) plane with one vacancy. The small balls are $\mathrm{H}$ atoms, which are inserted in the "internal surface" regions of the vacancy (spherical, yellow regions). (b) and (c) show the averaged number of adsorbed $\mathrm{H}$ atoms at the initial and the saddle states at $870 \mathrm{~K}$ in $\mathrm{Cu}-\mathrm{H}$ and $\mathrm{Pd}-\mathrm{H}$ systems, respectively. (d) manifests the hydrogen Gibbs excess as computed. The error bars in $(b-d)$ are the standard error of the data.

constant temperature of $870 \mathrm{~K}$. Figure $3(\mathrm{~b}, \mathrm{c})$ reveals that the $\Gamma_{\mathrm{H}}^{*}$ in the saddle-state is larger than the $\Gamma_{\mathrm{H}}^{0}$ in the ground-state for the $\mathrm{Cu}-\mathrm{H}$ and $\mathrm{Pd}-\mathrm{H}$ systems, that is, $\Gamma_{\mathrm{H}}^{\mathrm{m}}>0$ (Figure $3(\mathrm{~d})$ ), which is consistent with the results of PMF method with EAM potentials. We have confirmed that ignoring the thermal expansion of lattice and the thermal movements of metal atoms does not change the above conclusion, by performing additional simulations considering these effects (see also Figure S1 and Sec. S5 in the Supporting Information).

In order to more directly demonstrate the dynamical process of diffusion of vacancies, we performed a series of MD simulations with EAM potentials at $870 \mathrm{~K}$ and at different $\mathrm{H}$ fractions ranging from 0 to 0.3 for $\mathrm{Cu}-\mathrm{H}$ and $\mathrm{Pd}-\mathrm{H}$ systems (see Sec. S6 in Supporting Information). The results of the vacancy diffusion coefficient ratios with and without $\mathrm{H}, \mathrm{D}_{\mathrm{CuH}}^{\mathrm{V}} /$ $D_{\mathrm{Cu}}^{\mathrm{V}}$ and $D_{\mathrm{PdH}}^{\mathrm{V}} / D_{\mathrm{Pd}}^{\mathrm{V}}$, are also shown in Figure $2(\mathrm{a}, \mathrm{b})$, respectively. We can see that the $\mathrm{H}$-enhanced vacancy diffusion from the direct MD simulations is in good agreement with those from the PMF method. A direct comparison of the vacancy diffusion under the higher and the lower $\mathrm{H}$ concentrations is shown in the Supporting Information, 
Video S1. The scenario of chemical composition variation along the vacancy diffusion pathway is also observed in the direct $\mathrm{MD}$ simulations. A single vacancy diffusion event in the $\mathrm{Cu}-\mathrm{H}$ system at $870 \mathrm{~K}$ and $c_{\mathrm{H}}=0.1$ is shown in the Supporting Information, Video S2, as an example. We can see from the animation that two $\mathrm{H}$ atoms are adsorbed near the vacancy center of the initial state before the vacancy jump. After two additional $\mathrm{H}$ atoms appear near the vacancy center of the final state accidentally, the vacancy diffusion occurs. When approaching the saddle state, one of the $\mathrm{H}$ atoms escapes from the vacancy center of the initial state. Thus, a total of three $\mathrm{H}$ atoms are adsorbed near the vacancy in the saddle-point state.

Here, we apply the Gibbs adsorption isotherm ${ }^{34}$ in the $\mathrm{H}$ vacancy system to illuminate the relationship between the $\mathrm{H}$ enhanced vacancy diffusivity and the chemical composition excess in $\mathrm{H}$ at the initial and the saddle-point states. Following the Gibbsian concept, integrating eq 2 at constant $P$ and $T$ gives $\Delta G^{\mathrm{m}}=-\int_{-\infty}^{\mu_{\mathrm{H}}} \Gamma_{\mathrm{H}}^{\mathrm{m}} \mathrm{d} \mu_{\mathrm{H}}=-\int_{-\infty}^{\mu_{\mathrm{H}}}\left(\Gamma_{\mathrm{H}}^{*}-\Gamma_{\mathrm{H}}^{0}\right) \mathrm{d} \mu_{\mathrm{H}}$, where the chemical potential of $\mathrm{H}$ at $-\infty$ corresponds to pure metals with $\Gamma_{\mathrm{H}}^{*}=\Gamma_{\mathrm{H}}^{0}=0$. Based on TST and a mean field approximation in lattice gas models ${ }^{35}$ (see Sec. S7 in the Supporting Information), the vacancy diffusion coefficient ratio can be written as $D_{\mathrm{MH}}^{\mathrm{V}} / D_{\mathrm{M}}^{\mathrm{V}}=\exp \left(-\Delta G^{\mathrm{m}} / k_{\mathrm{B}} T\right)$ and

$$
\begin{aligned}
\Delta G^{\mathrm{m}}= & -\Gamma_{\text {sat }}^{*}\left[W\left(\frac{\Gamma_{\mathrm{H}}^{*}}{\Gamma_{\text {sat }}^{*}}\right)^{2}+k_{\mathrm{B}} T \ln \frac{\Gamma_{\text {sat }}^{*}}{\left(\Gamma_{\text {sat }}^{*}-\Gamma_{\mathrm{H}}^{*}\right)}\right] \\
& +\Gamma_{\text {sat }}^{0}\left[W\left(\frac{\Gamma_{\mathrm{H}}^{0}}{\Gamma_{\text {sat }}^{0}}\right)^{2}+k_{\mathrm{B}} T \ln \frac{\Gamma_{\text {sat }}^{0}}{\left(\Gamma_{\text {sat }}^{0}-\Gamma_{\mathrm{H}}^{0}\right)}\right]
\end{aligned}
$$

where $W$ is an effective $\mathrm{H}-\mathrm{H}$ interaction parameter, and $\Gamma_{\text {sat }}^{0}$ and $\Gamma_{\text {sat }}^{*}$ are, respectively, the saturation values of $\Gamma_{\mathrm{H}}^{0}$ and $\Gamma_{\mathrm{H}}^{*}$. The values of $W, \Gamma_{\text {sat }}^{0}$ and $\Gamma_{\text {sat }}^{*}$ can be determined by fitting the measured values of $\Gamma_{\mathrm{H}}^{0}, \Gamma_{\mathrm{H}}^{*}$, and $\Delta G^{\mathrm{m}}$ in the PMF calculations. These fitted results gives a comparable result of $D_{\mathrm{MH}}^{\mathrm{V}} / D_{\mathrm{M}}^{\mathrm{V}}$ with those from PMF, as shown in Figure 2(a,b). When $\mu_{\mathrm{H}} \gg \bar{\mu}_{\mathrm{H}}^{0}$ and $\mu_{\mathrm{H}} \gg \bar{\mu}_{\mathrm{H}}^{*}$, where $\bar{\mu}_{\mathrm{H}}^{0}$ and $\bar{\mu}_{\mathrm{H}}^{*}$ are, respectively, the standard values of the chemical potential at the ground and the saddlepoint states, $\mathrm{d}\left(\Delta G^{\mathrm{m}}\right) / \mathrm{d} \mu_{\mathrm{H}}=-\Gamma_{\mathrm{H}}^{\mathrm{m}} \rightarrow-\left(\Gamma_{\text {sat }}^{*}-\Gamma_{\text {sat }}^{0}\right)$, which means that $\Delta G^{\mathrm{m}}$ decreases linearly with increasing $\mu_{\mathrm{H}}$. Thus, $\Delta G^{\mathrm{m}} \approx-\left(\Gamma_{\text {sat }}^{*}-\Gamma_{\text {sat }}^{0}\right) \Delta \mu_{\mathrm{H}}$ and an activation excess, $\Gamma_{\mathrm{H}}^{\mathrm{m}}=\Gamma_{\text {sat }}^{*}$ - $\Gamma_{\text {sat }}^{0}$, can be defined to characterize the "hydrogen lubrication" effect at high $\mu_{\mathrm{H}}$. The values of $\Gamma_{\mathrm{H}}^{\mathrm{m}}$ are estimated to be 1.2 and 1.1 for $\mathrm{Cu}$ and $\mathrm{Pd}$, respectively, based on the EAM potentials. Compared to the values of $\Gamma_{\mathrm{H}}^{\mathrm{m}}$ at high $\mathrm{H}$ concentrations using the $a b$ initio GCMC (Figure 3(d)), we can approximately determine the $\Gamma_{\mathrm{H}}^{\mathrm{m}} \approx 1 \mathrm{H}$ at high $\mu_{\mathrm{H}}$ for $\mathrm{Cu}$ and $\mathrm{Pd}$.

We further demonstrated that the strong dragging effect of $\mathrm{H}$ on the vacancy diffusion given by the previous DFT calculations ${ }^{17,18}$ can be avoided using the $\mathrm{Cu}-\mathrm{H}$ system as an example through introducing the chemical composition variation of $\mathrm{H}$ along the diffusion pathway. At $0 \mathrm{~K}$, the vacancy adsorbs $\mathrm{H}$ atoms from the $\mathrm{H}$-environments as much as possible given that the Gibbs free-energy decreases (see Sec. S8 in the Supporting Information). Figure 4 shows the Gibbs free-energy along the diffusion pathway of a few $\mathrm{H}$-vacancies complex in $\mathrm{Cu}$ computed from DFT using the Gibbs freeenergy of a vacancy in pure $\mathrm{Cu}$ as a reference value. From Figure 4, we can see that the energy barrier for a vacancy without $\mathrm{H}\left(\mathrm{V}_{\mathrm{H}_{0}}\right)$ is about $0.72 \mathrm{eV}$. After minimization of the Gibbs free-energy of the vacancy, six $\mathrm{H}$ atoms occupy all the

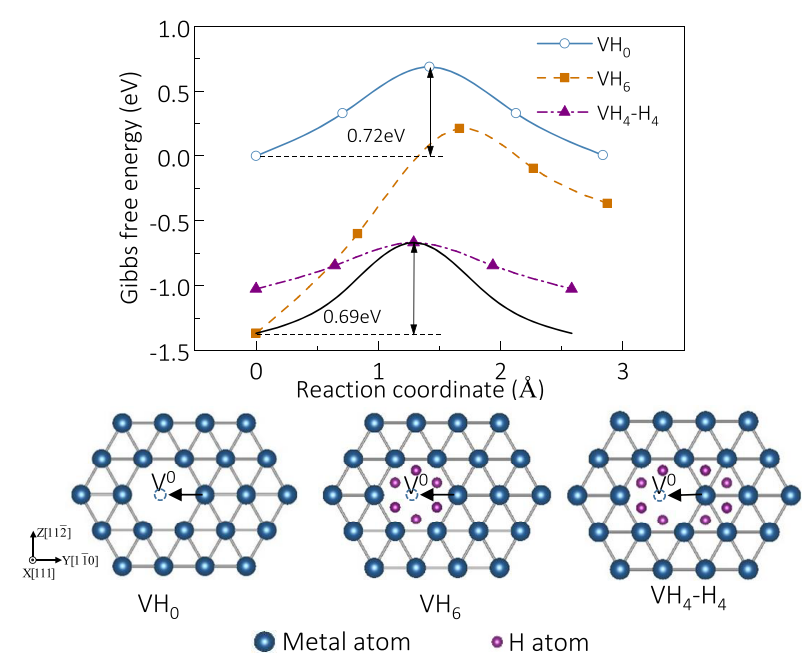

Figure 4. Gibbs free-energy as a function of reaction coordinate of the vacancy complex diffusion in $\mathrm{Cu}$ at $0 \mathrm{~K}$ computed using DFT. The below insets show the configurations of the fixed $\mathrm{H}$-position, and the arrows show the jump direction of one $\mathrm{Cu}$ atom. The black line indicates the diffusion pathway considering the chemical composition variation of $\mathrm{H}$ along the vacancy diffusion pathway.

$2 \mathrm{NN}(\mathrm{O})$ sites near the vacancy. When we fix the distribution of $\mathrm{H}$ over the diffusion process $\left(\mathrm{VH}_{6}\right)$, the energy barrier is as high as $1.58 \mathrm{eV}$. In contrast, the energy barrier can decrease to $0.69 \mathrm{eV}$ only if the saddle state evolves to the atomic configurations that eight $\mathrm{H}$ atoms are adsorbed near the vacancy of both the initial and the final states $\left(\mathrm{VH}_{4}-\mathrm{H}_{4}\right)$ to minimize the Gibbs free energy. Notably, although the chemical composition variation is demonstrated using the initial and the saddle states, the composition variation along the diffusion pathway is continuous (as qualitatively indicated by the black line in Figure 4).

The "hydrogen lubrication" effect on vacancy becomes more significant in higher $\mathrm{H}$-concentration environments (e.g., $c_{\mathrm{H}}>$ 0.01 , see Figure $2(\mathrm{a}, \mathrm{b}))$. The H-concentration in metals $\left(c_{\mathrm{H}} \equiv\right.$ $[\mathrm{H}] /[\mathrm{M}])$ can reach as high as $0.1 \sim 1.0$ in the high $\mathrm{H}_{2}$ pressure and high temperature experiments, e.g., $\mathrm{Pd}, \mathrm{Ni}$, and $\mathrm{Co}$ with an FCC structure at $P_{\mathrm{H}_{2}}=2.4-7.4 \mathrm{GPa}$ and $T=930-1350 \mathrm{~K}^{36}$ However, the vacancy concentration $\left(c_{\mathrm{V}} \equiv[\mathrm{V}] /[\mathrm{M}]\right)$ in metals can be also as high as 0.1 through forming superabundant vacancies, ${ }^{37}$ so whether there are still a sufficient number of $\mathrm{H}$ atoms that can be adsorbed by individual vacancies under experimental conditions needed to be considered. In the Pd$\mathrm{H}$ system, $c_{\mathrm{V}}$ is very small for $c_{\mathrm{H}}<0.6$ and increases linearly with increasing $c_{\mathrm{H}}$ until $c_{\mathrm{V}}=0.12$ at $c_{\mathrm{H}}=1$ based on lattice contraction measurements, and $c_{\mathrm{H}} / c_{\mathrm{V}}>8.3$ in the whole $c_{\mathrm{H}}$ range. ${ }^{36}$ In the $\mathrm{Nb}-\mathrm{H}$ system, $c_{\mathrm{H}} / c_{\mathrm{V}}$ was found to be about 78 for $c_{\mathrm{H}}<0.5$ by electric resistivity measurement. ${ }^{38}$ Since the maximum number of $\mathrm{H}$ atoms trapped in vacancy ${ }^{39,40}$ is about 6 , and the $\Gamma_{\mathrm{H}}^{\mathrm{m}} \approx 1 \mathrm{H}$, every vacancy in the $\mathrm{Pd}-\mathrm{H}$ and the $\mathrm{Nb}-$ $\mathrm{H}$ systems can easily access sufficient $\mathrm{H}$ atoms so that "hydrogen lubrication" can take effect. In the $\mathrm{Ni}-\mathrm{H}, \mathrm{Co}-\mathrm{H}$, and $\mathrm{Fe}-\mathrm{H}$ systems, $c_{\mathrm{H}} / c_{\mathrm{V}}$ were found to be $2.4,2.6$, and 3.4, respectively, for $c_{\mathrm{H}}<0.8$ through measuring the lattice contraction. $^{36,41}$ Thus, we expect there is sufficient hydrogen to drive the "hydrogen lubrication" effect under many experimental circumstances.

The activation excess caused by the chemical composition variation along the transition pathway should also affect the 
kinetics of other defect motion under the $\mathrm{H}$-environment, such as dislocation sliding by kink migration, grain boundary migration, and crack propagation, which also have higher capacity for attracting $\mathrm{H}$ due to positive activation volume (excess free volume) at the migration saddle-point. In addition to the above-mentioned classical mechanics treatment of $\mathrm{H}$ atoms, the quantum-dynamics effect of $\mathrm{H}^{42}$ should be considered to determine the distribution of $\mathrm{H}$ along the vacancy diffusion pathway at an extremely low temperature, which is beyond the scope of this paper.

In conclusion, the present work addresses the effect of $\mathrm{H}$ on the vacancy diffusivity in FCC metals. We find that $\mathrm{H}$ atoms can enhance the diffusivity of vacancy due to a chemical composition variation along the vacancy diffusion pathway. An additional activation parameter beyond the well-known activation volume and activation entropy, the activation excess, is derived and can be used to characterize the migration frequency of lattice defects embedded in $\mathrm{H}$-environments. If the activation excess $\Gamma_{\mathrm{H}}^{\mathrm{m}}=1 \mathrm{H}$, for every $10 \times$ increase in equivalent hydrogen gas partial pressure (fugacity) $P_{\mathrm{H}_{2}}$, we would predict a $3.162 \times$ increase in vacancy mobility, regardless of temperature. We term such hydrogen-dependent defect mobility the "hydrogen lubrication" effect. The concept of a hydrogen Gibbs excess on the saddle state can be applied to other interstitial or substitutional solutes and to the other thermally activated processes of materials in environments containing $\mathrm{O}, \mathrm{He}, \mathrm{N}, \mathrm{C}$, and so on. This triplet of thermodynamic quantities, the Gibbsian activation excess, along with the more well-known activation volume and activation entropy (Meyer-Neldel compensation rule) form the complete characterization of mobile defects and shed light on the fundamental kinetics of defects of materials in service in complex chemical environments.

\section{ASSOCIATED CONTENT}

\section{SI Supporting Information}

The Supporting Information is available free of charge at https://pubs.acs.org/doi/10.1021/acs.jpclett.0c01798.

Supporting video captions, relationship between the vacancy mobility and the hydrogen Gibbs excess, computational details of free-energy barrier calculations based on the potential-of-mean-force method, absorbed $\mathrm{H}$ atoms at initial and saddle states, $a b$ initio grand canonical Monte Carlo simulations, molecular dynamics simulations, mean field approximation in lattice gas model, Gibbs free energy using DFT at $0 \mathrm{~K}$ for $\mathrm{Cu}-\mathrm{H}$, construction of the EAM potential for $\mathrm{Cu}-\mathrm{H}$ (PDF) Video S1: Vacancy diffusion in $\mathrm{Cu}$ with $c_{\mathrm{H}}=0.005$ (left) and $c_{\mathrm{H}}=0.1$ (right) at $870 \mathrm{~K}$ for $10 \mathrm{~ns}$ using $\mathrm{MD}$ simulations (MP4)

Video S2: The $\mathrm{H}$-vacancy complex diffusion in $\mathrm{Cu}$ at $870 \mathrm{~K}$ and $c_{\mathrm{H}}=0.1$ from MD simulations (MP4)

\section{AUTHOR INFORMATION}

\section{Corresponding Authors}

Ju Li - Department of Nuclear Science and Engineering and Department of Materials Science and Engineering, Massachusetts Institute of Technology, Cambridge, Massachusetts 02139, United States; 이이.org/0000-00027841-8058; Email: liju@mit.edu

Shigenobu Ogata - Department of Mechanical Science and Bioengineering, Osaka University, Osaka 560-8531, Japan;
Center for Elements Strategy Initiative for Structural Materials, Kyoto University, Kyoto 606-8501, Japan; Email: ogata@ me.es.osaka-u.ac.jp

\section{Authors}

Jun-Ping Du - Center for Elements Strategy Initiative for Structural Materials, Kyoto University, Kyoto 606-8501, Japan; Department of Mechanical Science and Bioengineering, Osaka University, Osaka 560-8531, Japan

W. T. Geng - Department of Mechanical Science and Bioengineering, Osaka University, Osaka 560-8531, Japan; University of Science and Technology Beijing, Beijing 100083, China; (1) orcid.org/0000-0002-9838-5644

Kazuto Arakawa - Next Generation TATARA Co-Creation Centre, Organization for Industrial Innovation, Shimane University, Matsue 690-8504, Japan

Complete contact information is available at:

https://pubs.acs.org/10.1021/acs.jpclett.0c01798

\section{Notes}

The authors declare no competing financial interest.

\section{ACKNOWLEDGMENTS}

This work was supported by the Element Strategy Initiative for Structural Materials (ESISM) of MEXT, Grant Number JPMXP0112101000. Part of the calculations were performed on the large-scale computer systems at the Cybermedia Center, Osaka University. S.O. acknowledges the support by the JSPS KAKENHI Grant Nos. JP18H05450, JP18H05453, JP17H01238, and JP17K18827. W.T.G. thanks the NSFC (Grant Number U1760203) for support. J.L. acknowledges support by the U.S. Department of Energy (DOE) Fuel Cell Technologies Office under award number DE-EE0008830.

\section{REFERENCES}

(1) Pundt, A.; Kirchheim, R. Hydrogen in Metals: Microstructural Aspects. Annu. Rev. Mater. Res. 2006, 36, 555-608.

(2) Jeon, K.-J.; Moon, H. R.; Ruminski, A. M.; Jiang, B.; Kisielowski, C.; Bardhan, R.; Urban, J. J. Air-Stable Magnesium Nanocomposites Provide Rapid and High-Capacity Hydrogen Storage without Using Heavy-Metal Catalysts. Nat. Mater. 2011, 10, 286-290.

(3) Yoon, H.; Choi, M.; Lim, T.-W.; Kwon, H.; Ihm, K.; Kim, J. K.; Choi, S.-Y.; Son, J. Reversible Phase Modulation and Hydrogen Storage in Multivalent $\mathrm{VO}_{2}$ Epitaxial Thin Films. Nat. Mater. 2016, 15, 1113-1119.

(4) Christofides, C.; Mandelis, A. Solid-state Sensors for Trace Hydrogen Gas Detection. J. Appl. Phys. 1990, 68, R1-R30.

(5) Schmid, G. Large Clusters and Colloids. Metals in the Embryonic State. Chem. Rev. 1992, 92, 1709-1727.

(6) Sen, P.; Alam, K.; Das, T.; Banerjee, R.; Chakraborty, S. Combinatorial Design and Computational Screening of TwoDimensional Transition Metal Trichalcogenide Monolayers: Toward Efficient Catalysts for Hydrogen Evolution Reaction. J. Phys. Chem. Lett. 2020, 11, 3192-3197.

(7) Troiano, A. R. The Role of Hydrogen and Other Interstitials in the Mechanical Behavior of Metals. Metallogr., Microstruct., Anal. 2016, 5, 557-569.

(8) Nagumo, M. Hydrogen Related Failure of Steels - a New Aspect. Mater. Sci. Technol. 2004, 20, 940-950.

(9) Neeraj, T.; Srinivasan, R.; Li, J. Hydrogen Embrittlement of Ferritic Steels: Observations on Deformation Microstructure, Nanoscale Dimples and Failure by Nanovoiding. Acta Mater. 2012, 60, $5160-5171$. 
(10) Xie, D.-G.; Wang, Z.-J.; Sun, J.; Li, J.; Ma, E.; Shan, Z.-W. In Situ Study of the Initiation of Hydrogen Bubbles at the Aluminium Metal/Oxide Interface. Nat. Mater. 2015, 14, 899-903.

(11) Geng, W. T.; Wan, L.; Du, J.-P.; Ishii, A.; Ishikawa, N.; Kimizuka, H.; Ogata, S. Hydrogen Bubble Nucleation in $\alpha$-Iron. Scr. Mater. 2017, 134, 105-109.

(12) Hou, J.; Kong, X.-S.; Wu, X.; Song, J.; Liu, C. S. Predictive Model of Hydrogen Trapping and Bubbling in Nanovoids in Bcc Metals. Nat. Mater. 2019, 18, 833-839.

(13) Hänggi, P.; Talkner, P.; Borkovec, M. Reaction-Rate Theory: Fifty Years after Kramers. Rev. Mod. Phys. 1990, 62, 251-341.

(14) Kirkendall, E. O. Diffusion of Zinc in Alpha Brass. Trans. Metall. Soc. AIME 1942, 147, 104-110.

(15) Smigelskas, A. D.; Kirkendall, E. O. Zinc Diffusion in Alpha

Brass. Trans. Metall. Soc. AIME 1947, 171, 130-142.

(16) Myers, S. M.; Baskes, M. I.; Birnbaum, H. K.; Corbett, J. W.; Deleo, G. G.; Estreicher, S. K.; Haller, E. E.; Jena, P.; Johnson, N. M.; Kirchheim, R.; Pearton, S. J.; Stavola, M. J. Hydrogen Interactions with Defects in Crystalline Solids. Rev. Mod. Phys. 1992, 64, 559-617.

(17) Wang, Y.; Connétable, D.; Tanguy, D. Hydrogen Influence on Diffusion in Nickel from First-Principles Calculations. Phys. Rev. B: Condens. Matter Mater. Phys. 2015, 91, No. 094106.

(18) Hayward, E.; Fu, C. C. Interplay between Hydrogen and Vacancies in $\alpha$-Fe. Phys. Rev. B: Condens. Matter Mater. Phys. 2013, 87, 174103.

(19) Kirchheim, R. Reducing Grain Boundary, Dislocation Line and Vacancy Formation Energies by Solute Segregation. I. Theoretical Background. Acta Mater. 2007, 55, 5129-5138.

(20) Kirchheim, R. Reducing Grain Boundary, Dislocation Line and Vacancy Formation Energies by Solute Segregation: II. Experimental Evidence and Consequences. Acta Mater. 2007, 55, 5139-5148.

(21) Zhu, T.; Li, J. Ultra-Strength Materials. Prog. Mater. Sci. 2010, $55,710-757$.

(22) Hara, S.; Li, J. Adaptive Strain-Boost Hyperdynamics Simulations of Stress-Driven Atomic Processes. Phys. Rev. B: Condens. Matter Mater. Phys. 2010, 82, 184114.

(23) Li, Q.-J.; Xu, B.; Hara, S.; Li, J.; Ma, E. Sample-Size-Dependent Surface Dislocation Nucleation in Nanoscale Crystals. Acta Mater. 2018, 145, 19-29.

(24) Den Otter, W. K.; Briels, W. J. Free Energy from Molecular Dynamics with Multiple Constraints. Mol. Phys. 2000, 98, 773-781.

(25) Sugimoto, H.; Fukai, Y. Solubility of Hydrogen in Metals under High Hydrogen Pressures: Thermodynamical Calculations. Acta Metall. Mater. 1992, 40, 2327-2336.

(26) Fukai, Y. Hydrogen-Induced Superabundant Vacancies in Metals: Implication for Electrodeposition. Defect Diffus. Forum 2011, 312-315, 1106-1115.

(27) Baranowski, B.; Majchrzak, S.; Flanagan, T. B. The Volume Increase of Fcc Metals and Alloys Due to Interstitial Hydrogen over a Wide Range of Hydrogen Contents. J. Phys. F: Met. Phys. 1971, 1, 258-261.

(28) Fukumuro, N.; Yokota, M.; Yae, S.; Matsuda, H.; Fukai, Y. Hydrogen-Induced Enhancement of Atomic Diffusion in Electrodeposited Pd Films. J. Alloys Compd. 2013, 580, S55-S57.

(29) Mishin, Y.; Mehl, M. J.; Papaconstantopoulos, D. A.; Voter, A. F.; Kress, J. D. Structural Stability and Lattice Defects in Copper: Ab Initio, Tight-Binding, and Embedded-Atom Calculations. Phys. Rev. B: Condens. Matter Mater. Phys. 2001, 63, 224106.

(30) Du, J. P.; Wang, C. Y.; Yu, T. Construction and Application of Multi-Element EAM Potential (Ni-Al-Re) in $\gamma / \gamma^{\prime}$ Ni-Based Single Crystal Superalloys. Modell. Simul. Mater. Sci. Eng. 2013, 21, No. 015007

(31) Zhou, X. W.; Zimmerman, J. A.; Wong, B. M.; Hoyt, J. J. An Embedded-Atom Method Interatomic Potential for Pd-H Alloys. J. Mater. Res. 2008, 23, 704-718.

(32) Mehrer, H. Diffusion in Solids: Fundamentals, Methods, Materials, Diffusion-Controlled Processes; Springer: Berlin, 2007.
(33) Wexler, R. B.; Qiu, T.; Rappe, A. M. Automatic Prediction of Surface Phase Diagrams Using Ab Initio Grand Canonical Monte Carlo. J. Phys. Chem. C 2019, 123, 2321-2328.

(34) Chattoraj, D. K.; Birdi, K. S. Adsorption and the Gibbs Surface Excess; Plenum: New York, 1984.

(35) Kirchheim, R.; Somerday, B.; Sofronis, P. Chemomechanical Effects on the Separation of Interfaces Occurring during Fracture with Emphasis on the Hydrogen-Iron and Hydrogen-Nickel System. Acta Mater. 2015, 99, 87-98.

(36) Harada, S.; Yokota, S.; Ishii, Y.; Shizuku, Y.; Kanazawa, M.; Fukai, Y. A Relation between the Vacancy Concentration and Hydrogen Concentration in the $\mathrm{Ni}-\mathrm{H}, \mathrm{Co}-\mathrm{H}$ and $\mathrm{Pd}-\mathrm{H}$ Systems. $J$. Alloys Compd. 2005, 404-406, 247-251.

(37) Fukai, Y.; Ōkuma, N. Formation of Superabundant Vacancies in Pd Hydride under High Hydrogen Pressures. Phys. Rev. Lett. 1994, 73, 1640-1643.

(38) Koike, H.; Shizuku, Y.; Yazaki, A.; Fukai, Y. Superabundant Vacancy Formation in $\mathrm{Nb}-\mathrm{H}$ Alloys; Resistometric Studies. J. Phys.: Condens. Matter 2004, 16, 1335-1349.

(39) Fukai, Y. Hydrogen-Induced Enhancement of Atomic Diffusion in Metals. Defect Diffus. Forum 2010, 297-301, 132-141.

(40) Nazarov, R.; Hickel, T.; Neugebauer, J. Ab Initio Study of HVacancy Interactions in Fcc Metals: Implications for the Formation of Superabundant Vacancies. Phys. Rev. B: Condens. Matter Mater. Phys. 2014, 89, 144108.

(41) Hiroi, T.; Fukai, Y.; Mori, K. The Phase Diagram and Superabundant Vacancy Formation in $\mathrm{Fe}-\mathrm{H}$ Alloys Revisited. J. Alloys Compd. 2005, 404-406, 252-255.

(42) Kimizuka, H.; Ogata, S.; Shiga, M. Unraveling Anomalous Isotope Effect on Hydrogen Diffusivities in Fcc Metals from First Principles Including Nuclear Quantum Effects. Phys. Rev. B: Condens. Matter Mater. Phys. 2019, 100, No. 024104. 